\title{
Different expression of $\beta$ subunits of the KCa1.1 channel by invasive and non- invasive human fibroblast-like synoviocytes
}

Zoltán Pethő'1,2, Mark R. Tanner ${ }^{1,3}$, Rajeev B. Tajhya ${ }^{1,4}$, Redwan Huq ${ }^{1,4}$, Teresina Laragione ${ }^{5}$, Gyorgy Panyi ${ }^{2}$, Pércio S. Gulko ${ }^{5}$ and Christine Beeton ${ }^{1,6,7^{*}}$

\begin{abstract}
Background: Fibroblast-like synoviocytes (FLS) in rheumatoid arthritis (RA-FLS) contribute to joint inflammation and damage characteristic of the disease. RA-FLS express KCa1.1 (BK, Slo1, MaxiK, KCNMA1) as their major plasma membrane potassium channel. Blocking KCa1.1 reduces the invasive phenotype of RA-FLS and attenuates disease severity in animal models of RA. This channel has therefore emerged as a promising therapeutic target in RA. However, the pore-forming a subunit of KCa1.1 is widely distributed in the body, and blocking it induces severe side effects, thus limiting its value as a therapeutic target. On the other hand, KCa1.1 channels can also contain different accessory subunits with restricted tissue distribution that regulate channel kinetics and pharmacology. Identification of the regulatory subunits of KCa1.1 expressed by RA-FLS may therefore provide the opportunity for generating a selective target for RA treatment.

Methods: Highly invasive RA-FLS were isolated from patients with RA, and FLS from patients with osteoarthritis $(\mathrm{OA})$ were used as minimally invasive controls. The $\beta$ subunit expression by FLS was assessed by quantitative reverse transcription polymerase chain reactions, Western blotting, and patch-clamp electrophysiology combined with pharmacological agents. FLS were sorted by flow cytometry on the basis of their CD44 expression level for comparison of their invasiveness and with their expression of KCa1.1 $a$ and $\beta$ subunits. $\beta 1$ and $\beta 3$ subunit expression was reduced with small interfering RNA (siRNA) to assess their specific role in KCa1.1 a expression and function and in FLS invasiveness.
\end{abstract}

Results: We identified functional $\beta 1$ and $\beta 3 \mathrm{~b}$ regulatory subunits in RA-FLS. KCa1.1 $\beta 3 \mathrm{~b}$ subunits were expressed by $70 \%$ of the cells and were associated with highly invasive CD44 high RA-FLS, whereas minimally invasive CD44 ${ }^{\text {low }}$ RA-FLS and OA-FLS expressed either $\beta 1$ subunit. Furthermore, we found that silencing the $\beta 3$ but not the $\beta 1$ subunit with siRNA reduced KCa1.1 channel density at the plasma membrane of RA-FLS and inhibited RA-FLS invasiveness.

Conclusions: Our findings suggest the KCa1.1 channel composed of a and $\beta 3 \mathrm{~b}$ subunits as an attractive target for the therapy of RA.

Keywords: Arthritis, Autoimmune disease, Cell migration, Electrophysiology, Patch clamp, Potassium channel, Regulatory subunit, Synovial fibroblast

\footnotetext{
* Correspondence: beeton@bcm.edu

'Department of Molecular Physiology and Biophysics, Mail Stop BCM335,

Room S409A, Baylor College of Medicine, Houston, TX 77030, USA

${ }^{6}$ Biology of Inflammation Center, Baylor College of Medicine, Houston, TX

77030, USA

Full list of author information is available at the end of the article
} 


\section{Background}

Rheumatoid arthritis (RA) is a chronic and systemic autoimmune disease that predominantly involves diarthrodial joints but can also target vital internal organs, including the heart and lungs [1-3]. In the last decades, management of RA has significantly improved with the development of new treatments; however, disease remission is rarely achieved, and most patients attain only mild to modest improvement [4]. Furthermore, current therapies significantly impair immune responses, rendering patients more susceptible to infections and cancer [5]. Therefore, novel therapeutic options that lead to pronounced improvement or remission without inducing immunosuppression are needed.

Fibroblast-like synoviocytes (FLS) in RA (RA-FLS) have been implicated in disease pathogenesis, as they exhibit a transformed, "tumor-like" phenotype with increased invasiveness and production of proteases and of various proinflammatory and proangiogenic factors [6-8]. Importantly, the ex vivo invasiveness of FLS correlates with radiographic and histologic damage and therefore with disease severity $[9,10]$.

Highly invasive RA-FLS and FLS from arthritic rats express the functional calcium-activated potassium channel KCa1.1 (also known as BK, Slo1, MaxiK, or KCNMA1) as the major $\mathrm{K}^{+}$channel at their plasma membrane [11, 12]. Blocking the function of KCa1.1 pore-forming $\alpha$ subunits in these FLS with paxilline inhibits their invasiveness and stops disease progression in animal models of RA $[11,12]$. However, paxilline is a lipophilic small molecule that blocks all KCa1.1 channels found in major organs, regardless of channel subunit composition [13-15], and can cross cell membranes as well as the blood-brain barrier. It therefore induces severe side effects, such as tremors, incontinence, and hypertension [16-18]. Despite the drawbacks of the unspecific block of $\mathrm{KCa} 1.1$ by paxilline, the channel remains an attractive target for therapy because the regulatory subunits of $\mathrm{KCa} 1.1$ have restricted tissue distribution and affect channel pharmacology $[19,20]$. Four $\beta$ subunits with distinct amino acid sequences have been described: $\beta 1$ (KCNMB1, UniProt identifier Q16558), found in smooth muscles; $\beta 2$ (KCNMB2, UniProt identifier Q9Y691), prevalent in the adrenal gland and brain; $\beta 3$ (KCNMB3, UniProt identifier Q9NPA1), expressed mainly in the testis; and B4 (KCNMB4, UniProt identifier Q86W47), mostly expressed in the central nervous system [20-26]. The presence of $\beta$ subunits affects the kinetics of the channel as well as the selectivity and affinity of various KCa1.1 channel modulators. Steroid compounds, such as lithocholic acid (LCA), selectively enhance KCa1.1 currents only if the channel contains the $\beta 1$ subunit $[27,28]$. In contrast, arachidonic acid (AA) amplifies currents in the presence of the $\beta 2$ or $\beta 3$ subunit but not the $\beta 1$ or $\beta 4$ subunit [29], whereas the scorpion toxins iberiotoxin and charybdotoxin (ChTX) fail to inhibit $\mathrm{KCa} 1.1$ in the presence of the $\beta 4$ subunit [21]. In addition, the $\beta 1, \beta 2$, and $\beta 4$ subunits modulate membrane translocation of the $\mathrm{KCa} 1.1$-conducting $\alpha$ subunit $[23,24,30]$. To date, however, no KCa1.1 $\beta$ subunit has been described in RA-FLS. Our aims in this study were to determine whether RA-FLS express any accessory $\beta$ subunits and whether expression of these subunits varies with the invasive potential of these cells.

\section{Methods}

\section{Ethical considerations}

FLS were collected and banked for research in a deidentified manner by the Feinstein Institute for Medical Research Tissue Donation Program (Manhasset, NY, USA) under the approval of the institutional review board (IRB) of the Feinstein Institute for Medical Research. All patients provided written consent to have their tissues, RNA, DNA, and cells studied. The consent forms are held by the Feinstein Institute Tissue Donation Program; the authors of this study had access only to cells and deidentified data. The IRB at Baylor College of Medicine has established that the work conducted there did not constitute human research, since the samples were already banked for research and were deidentified, and thus could not be traced back to their donors.

Rat testes were obtained from Prof. Robert M. Bryan's laboratory (Department of Anesthesiology, Baylor College of Medicine, Houston, TX, USA). Rats were killed following American Veterinary Medical Association guidelines under an institutional animal care and use committee-approved protocol at Baylor College of Medicine. Discarded testes from healthy animals were obtained as control samples for our studies.

\section{Cells}

FLS from 14 patients with RA (age $58 \pm 10$ years) and 4 patients with osteoarthritis (OA) (age $57 \pm 5$ years), defined according to the criteria of the American College of Rheumatology [31], were purchased from Asterand Bioscience (Detroit, MI, USA) or collected as described elsewhere [32, 33] (Table 1). Cells were used between passages 4 and 11 for all experiments. They were cultured in DMEM (Life Technologies, Grand Island, NY, USA) supplemented with $10 \mathrm{IU} / \mathrm{ml}$ penicillin, $0.1 \mathrm{~g} / \mathrm{ml}$ streptomycin, $1 \mathrm{mM}$ sodium pyruvate, $2 \mathrm{mg} / \mathrm{ml} \mathrm{L-glutamine,} \mathrm{and} 10 \% \mathrm{FBS}$.

\section{Quantitative reverse transcription-polymerase chain reactions}

Total RNA, isolated from cell pellets using TRIzol reagent (Life Technologies), was reverse-transcribed with 
Table 1 Characteristics of the subjects who donated fibroblast-like synoviocytes for this study

\begin{tabular}{|c|c|c|c|c|c|c|c|c|}
\hline Donor & Diagnosis & Sex & Ethnicity & $\begin{array}{l}\text { Disease duration } \\
\text { (years) }\end{array}$ & $\mathrm{RF}$ & Medications & Origin of cells & $\begin{array}{l}\text { Figures showing which cells } \\
\text { were used }^{\mathrm{a}}\end{array}$ \\
\hline RA-1 & RA & Female & White & 3 & + & NSAID & $\begin{array}{l}\text { Asterand } \\
\text { Bioscience }\end{array}$ & $1 a, b ; 2 a, b ; 3 a, b, d$ \\
\hline RA-2 & RA & Male & Hispanic & $<1$ & + & Prednisone, DMARD & $\begin{array}{l}\text { Asterand } \\
\text { Bioscience }\end{array}$ & $2 a, b ; 5 a, b, c ; 6 b$ \\
\hline RA-3 & RA & Female & White & $<1$ & + & NSAID & $\begin{array}{l}\text { Asterand } \\
\text { Bioscience }\end{array}$ & $2 a, b ; 3 a, b, d$ \\
\hline RA-4 & RA & Female & White & 12 & + & NSAID, prednisone & $\begin{array}{l}\text { Asterand } \\
\text { Bioscience }\end{array}$ & $1 a, b ; 2 a, b ; 4 c$ \\
\hline RA-5 & RA & Female & Hispanic & 2 & + & DMARD, NSAID & FITDP & $1 a, b ; 4 a, b, e, f, g ; 5 a, b, c$ \\
\hline RA- 6 & RA & Female & White & 21 & + & Prednisone, DMARD & FITDP & $3 a, b, c, d$ \\
\hline RA-7 & RA & Female & White & 30 & + & Prednisone, DMARD & FITDP & $2 a, b ; 3 a, b, c, d ; 4 c, d, e, f$ \\
\hline RA-8 & RA & Female & White & 20 & + & Etanercept, prednisone & FITDP & $1 a, b ; 2 a, b$ \\
\hline RA-9 & RA & Female & White & 21 & + & $\begin{array}{l}\text { Leflunomide, etanercept, } \\
\text { prednisone }\end{array}$ & FITDP & $1 a, b ; 4 a, b ; 5 a, b, c ; 6 a, b$ \\
\hline RA-10 & RA & Male & White & 15 & + & $\begin{array}{l}\text { NSAID, prednisone, } \\
\text { adalimumab }\end{array}$ & FITDP & $2 a, b ; 3 c ; 4 c, d, e, f, g$ \\
\hline RA-11 & RA & Female & $\begin{array}{l}\text { African } \\
\text { American }\end{array}$ & 11 & + & $\begin{array}{l}\text { Hydroxychloroquine, } \\
\text { prednisone }\end{array}$ & FITDP & $2 a, b ; 3 c ; 4 c, d, e, f, g$ \\
\hline RA-12 & RA & Male & White & $>10$ & + & Etanercept & FITDP & $1 a, b ; 3 c ; 4 a, b$ \\
\hline RA-13 & RA & Female & Hispanic & $>10$ & + & Methotrexate, prednisone & FITDP & $3 a, b, c, d ; 4 c, d, e, f ; 6 a, b$ \\
\hline RA-14 & RA & Female & White & 3 & + & Prednisone, methotrexate & FITDP & $5 a, b, c ; 6 a, b$ \\
\hline OA-1 & $\mathrm{OA}$ & Female & White & 12 & - & None & $\begin{array}{l}\text { Asterand } \\
\text { Bioscience }\end{array}$ & $4 c, d, e, f$ \\
\hline $\mathrm{OA}-2$ & $\mathrm{OA}$ & Male & White & 16 & - & None & $\begin{array}{l}\text { Asterand } \\
\text { Bioscience }\end{array}$ & $4 c, d, e, f$ \\
\hline $\mathrm{OA}-3$ & OA & Male & White & 25 & $\begin{array}{l}\mathrm{N} / \\
\mathrm{A}\end{array}$ & None & FITDP & $4 c, d, e, f$ \\
\hline OA-4 & $\mathrm{OA}$ & Male & White & 78 & $\begin{array}{l}\mathrm{N} / \\
\mathrm{A}\end{array}$ & None & FITDP & $4 c, d, e, f$ \\
\hline
\end{tabular}

DMARD disease-modifying antirheumatic drug, FITDP Feinstein Institute Tissue Donation Program, N/A not available, NSAID non-steroidal anti-inflammatory drug, $O A$ osteoarthritis, $R A$ rheumatoid arthritis, $R F$ rheumatoid factor

Patients with RA were $58 \pm 10$ years old, and patients with OA were $57 \pm 5$ years old

${ }^{a}$ Cross-references to figures in this article displaying which cells were used

SuperScript III reverse transcriptase and random hexamer primers (Life Technologies) according to the manufacturer's instructions. The resulting complementary DNA (cDNA) was used as a template for quantitative polymerase chain reaction (qPCR) primers (Table 2), which were designed from the National Institutes of Health qPrimerDepot (http://primerdepot.nci.nih.gov/) or designed manually and tested by PrimerBlast (http:// www.ncbi.nlm.nih.gov/tools/primer-blast/). Amplicon sizes were between 70 and $250 \mathrm{bp}$. qPCRs were conducted in final volumes of $10 \mu \mathrm{l}$ diluted cDNA (1:10) containing $4 \mu \mathrm{l}$ of oligo forward and reverse primers $(2.5 \mu \mathrm{M}$ each) and $6 \mu \mathrm{l}$ of iTAQ SYBR Green Supermix (Bio-Rad Laboratories, Hercules, CA, USA). Reactions were run in a ViiA 7 RealTime PCR System (Life Technologies). The cycling condition were 20 seconds at $95{ }^{\circ} \mathrm{C}, 40$ cycles at $95^{\circ} \mathrm{C}$ for $1 \mathrm{sec}$ ond and $60{ }^{\circ} \mathrm{C}$ for 20 seconds, $95{ }^{\circ} \mathrm{C}$ for 15 seconds, $60{ }^{\circ} \mathrm{C}$ for 1 minute, and a gradient from $60{ }^{\circ} \mathrm{C}$ to $95^{\circ} \mathrm{C}$ for $15 \mathrm{mi}-$ nutes. The results were analyzed using $\mathrm{ViiA}^{\mathrm{Tm}} 7$ software.

\section{Western blotting}

RA-FLS and a rat testis were lysed in radioimmunoprecipitation assay buffer (Sigma-Aldrich, St. Louis, MO, USA) containing $1 \%$ protease inhibitors. Protein levels were measured using the Bradford assay. Equal amounts of protein $(20 \mu \mathrm{g})$ were loaded and separated by SDSPAGE (Life Technologies), then transferred onto nitrocellulose membranes (Bio-Rad Laboratories) according to the manufacturer's instructions. Blots were incubated in overnight blocking solution consisting of $4 \%$ Blotto nonfat milk (Santa Cruz Biotechnology, Santa Cruz, CA, USA). Blots were probed using antibodies specific for the human KCa1.1 channel $\alpha$ and $\beta$ subunits (Table 3). Each primary antibody was diluted 1:500 in blocking 
Table 2 Primers used for quantitative polymerase chain reactions

\begin{tabular}{|c|c|c|c|c|}
\hline Subunit & Accession number & Sense (forward) 5'-3' & Antisense (reverse) 3'-5' & Base pair \\
\hline$a$ & NM_002247 & GCTCAAGTACCTGTGGACCG & CTGGTTTGAGAGTGCCATCC & 104 \\
\hline$\beta 1$ & NM_004137 & CTGTACCACACGGAGGACAC & GCTCTGACCTTCTCCACGTC & 107 \\
\hline$\beta 2 a$ & NM_181361 & ATTAAGCGTGGCTITTGAGG & GTTGGTCCAGGGTCTCCTIT & 98 \\
\hline$\beta 2 b$ & NM_005832 & GAGAAAGAGCAACAAAGCGG & TTAGCAAATCCCAGACATTGC & 107 \\
\hline$\beta 3 a$ & NM_171828 & AAATCACACTTCAGGGCAGC & GCACATCTAGTGGGTCTCCA & 105 \\
\hline$\beta 3 b$ & NM_171829 & TCTGAGTGTGAGGGGCTCTT & GCACATCTAGTGGGTCTCCA & 105 \\
\hline$\beta 3 c$ & NM_171830 & CCATGATGGGCTTCTCAGTC & GCAGTGCAGGTCGATTCTTC & 100 \\
\hline$\beta 3 d$ & NM_014407 & AGGGACGTGCAATATCCCTG & GAAAGGCTGTCCTGTGTCTCGT & 351 \\
\hline$\beta 3$ e & NM_001163677 & ACCCGTGTCTTCAGGTGTIT & TCTGTAACATCACGCTTGGGA & 107 \\
\hline$\beta 4$ & NM_014505 & CTGAGTCCAACTCTAGGGCG & GATाTCTCTCTTACAGGGAGGG & 96 \\
\hline GAPDH & NM_002046 & AAGGTGAAGGTCGGAGTCAA & AATGAAGGGGTCATTGATGG & 108 \\
\hline
\end{tabular}

GAPDH glyceraldehyde 3-phosphate dehydrogenase

solution. As loading controls, we used antiactin antibodies. Overnight incubation and washing were followed by a 2-h probing with IR-680- or IR-800-labeled secondary antibodies (Table 3 ) for $1 \mathrm{~h}$ at room temperature. The membranes were washed three times for 15 minutes with PBS $+0.1 \%$ Tween 20 . Visualization was performed with a LI-COR Odyssey Scanner (LI-COR Biosciences, Lincoln, NE, USA), and data were analyzed with ImageJ software (National Institutes of Health, Bethesda, MD, USA).

Table 3 Characteristics of the antibodies used for this study

\begin{tabular}{|c|c|c|c|c|c|}
\hline Target & Host & Vendor (catalog number; manufacturer location) & Conjugation & Clone & Use \\
\hline \multicolumn{6}{|l|}{ Primary antibodies } \\
\hline Actin & Rabbit & Sigma-Aldrich (A2066; St. Louis, MO, USA) & - & - & WB \\
\hline Actin & Mouse & Sigma-Aldrich (A3853) & - & AC-40 & WB \\
\hline Cadherin-11 & Mouse & $\begin{array}{l}\text { Thermo Fisher Scientific (MA1-06306; } \\
\text { Rockford, IL, USA) }\end{array}$ & - & $16 \mathrm{~A}$ & FC \\
\hline CD44 & Mouse & Abcam (ab187571; Cambridge, MA, USA) & Alexa Fluor 488 & MEM85 & FC, EP \\
\hline KCa1.1a & Mouse & $\begin{array}{l}\text { Antibodies, Inc. (NeuroMab, 75-022; } \\
\text { UC Davis/NIH NeuroMab Facility, Davis, CA, USA) }\end{array}$ & - & $L 6 / 60$ & WB \\
\hline KCa1.1a & Rabbit & EMD Millipore (AB5228; Billerica, MA, USA) & - & - & FC \\
\hline KCa1.1ß1 & Rabbit & $\begin{array}{l}\text { Novus Biologicals (NBP1-33484; } \\
\text { Littleton, CO, USA) }\end{array}$ & - & - & WB \\
\hline KCa1.1ß2 & Mouse & $\begin{array}{l}\text { Antibodies, Inc. (NeuroMab, } 75-087 ; \\
\text { UC Davis/NIH NeuroMab Facility) }\end{array}$ & - & N53/32 & WB \\
\hline KCa1.1 pan- $\beta 3$ & Rabbit & Abcam (ab137041) & - & EPR9543(B) & WB \\
\hline KCa1.1 $\beta 3 a, \beta c, \beta d, \beta e$ & Mouse & $\begin{array}{l}\text { Rockland Immunochemicals } \\
\text { (200-301-E96; Limerick, PA, USA) }\end{array}$ & - & S40B-18 & WB \\
\hline KCa1.1ß4 & Mouse & $\begin{array}{l}\text { Antibodies, Inc. (NeuroMab, 75-086; } \\
\text { UC Davis/NIH NeuroMab Facility) }\end{array}$ & - & $\mathrm{L} 18 \mathrm{~A} / 3$ & WB \\
\hline MMP-2 & Mouse & BioLegend (634802; San Diego, CA, USA) & - & F14P4D3 & $\mathrm{FC}$ \\
\hline Podoplanin & Rat & BioLegend (337003) & Phycoerythrin & NC-08 & FC \\
\hline \multicolumn{6}{|l|}{ Secondary antibodies } \\
\hline Mouse lgG & Goat & $\begin{array}{l}\text { LI-COR Biosciences } \\
\text { (926-32210; Lincoln, NE, USA) }\end{array}$ & Infrared-800 & - & WB \\
\hline Mouse lgG1 & Goat & Thermo Fisher Scientific (A-21127) & Alexa Fluor 555 & - & FC \\
\hline Rabbit lgG & Donkey & LI-COR Biosciences (926-68023) & Infrared-680 & - & WB \\
\hline Rabbit lgG & Goat & Abcam (ab150079) & Alexa Fluor 647 & - & FC \\
\hline
\end{tabular}

EP electrophysiology, $F C$ flow cytometry, IgG immunoglobulin G, $K C a$ calcium-activated potassium channel, MMP matrix metalloproteinase, WB Western blot analysis 


\section{Electrophysiology}

Cells were plated on glass coverslips and allowed to adhere. When indicated, cells were incubated for $15 \mathrm{mi}-$ nutes at room temperature with fluorophore-conjugated anti-CD44 antibodies (Table 3) and washed, and CD44 ${ }^{\text {high }}$ and CD $44^{\text {low }}$ cells were immediately assessed for $\mathrm{K}^{+}$currents [34]. Total $\mathrm{K}^{+}$currents were recorded using the patch-clamp technique in the whole-cell configuration as described previously [11, 12, 35]. The internal solution contained $10 \mathrm{mM}$ ethylene glycol tetraacetic acid, $5 \mathrm{mM}$ HEPES, and $5 \mu \mathrm{M}$ free $\mathrm{Ca}^{2+}$, calculated using Maxchelator (http://maxchelator.stanford.edu/CaEGTA-TS.htm). The external solution contained $160 \mathrm{mM} \mathrm{NaCl}, 4.5 \mathrm{mM} \mathrm{KCl}$, $2 \mathrm{mM} \mathrm{CaCl}_{2}, 1 \mathrm{mM} \mathrm{MgCl}_{2}$, and $10 \mathrm{mM}$ HEPES, $\mathrm{pH}$ 7.4. Experiments were performed at room temperature $\left(20-22{ }^{\circ} \mathrm{C}\right)$. FLS cell capacitances ranged from 9 to $17 \mathrm{pF}$. Results were analyzed using IGOR Pro software (WaveMetrics, Lake Oswego, OR, USA).

\section{Ion channel modulators}

LCA enhances KCa1.1 currents only when the channel contains the $\beta 1$ subunit $[28,36,37]$. A stock of LCA (Sigma-Aldrich) was prepared in 1:10 solution of dimethyl sulfoxide (DMSO) and $70 \%$ ethanol. AA enhances KCa1.1 currents only when the channel contains $\beta 2$ or $\beta 3$ subunits $[29,36]$. A stock of AA (Sigma-Aldrich) was prepared in DMSO. Paxilline blocks KCa1.1 channels, regardless of $\beta$ subunit expression [11, 14, 38]. A stock of paxilline (Fermentek, Jerusalem, Israel) was prepared in DMSO. ChTX blocks all KCa1.1 channels, unless they contain the $\beta 4$ subunit [21, 36, 39]. A stock of ChTX (Peptides International, Louisville, KY, USA) was prepared in P6N buffer $(10 \mathrm{mM} \mathrm{NaHPO}, 0.8 \% \mathrm{NaCl}, 0.05 \%$ Tween 20, $\mathrm{pH}$ 6.0) [40-42]. The stock solutions were further diluted with electrophysiology bath solution immediately before use so that final DMSO concentrations did not exceed $0.05 \%$.

\section{Flow cytometry}

Detection of the $\alpha$ subunit of KCa1.1 and of CD44, podoplanin, cadherin 11 , and intracellular matrix metalloproteinase (MMP)-2 was performed as previously described $[12,41,43]$ using antibodies listed in Table 3. Cells were treated with brefeldin A (eBioscience, San Diego, CA, USA) for $6 \mathrm{~h}$ before detection of intracellular MMP-2. They were fixed and permeabilized with $0.5 \%$ saponin for detection of intracellular epitopes. Data were acquired on a BD FACSCanto II flow cytometer (BD Biosciences, San Jose, CA, USA) using BD FACSDiva software and analyzed using FlowJo software (Treestar, Ashland, OR, USA). Alternatively, live $\mathrm{CD} 44^{\text {high }}$ and $\mathrm{CD} 44^{\text {low }}$ cells were sorted under sterile conditions using a FACSAria II flow cytometer (BD Biosciences) and immediately used for invasion assays.

\section{Small interfering RNA transfection}

RA-FLS were kept in serum-free medium for $24 \mathrm{~h}$ before small interfering RNA (siRNA) treatment with DharmaFECT siRNA transfection reagent and siRNA against GAPDH, KCNMB1, or KCNMB3 (GE Dharmacon, Lafayette, $\mathrm{CO}$ ) as described previously [12]. Cells were used 40-80 h later.

\section{Invasion assays}

The ex vivo invasiveness of FLS was assayed in a transwell system using collagen-rich, Matrigel-coated inserts (BD Biosciences) as described elsewhere [11, 12, 44].

\section{Statistical analysis}

We performed nonparametric one-way analysis of variance (Kruskal-Wallis test) to calculate the statistical significance of our results, followed by Dunn's post hoc test (Prism software; GraphPad Software, La Jolla, CA, USA). $p$ values less than 0.05 were considered significant.

\section{Results}

RA-FLS express multiple $\beta$ subunits at the messenger RNA and protein levels

Analysis of messenger RNA (mRNA) levels by quantitative reverse transcription-PCR (qRT-PCR) in RA-FLS showed expression of most KCa1.1 $\beta$ subunits (Fig. 1a), although the $\beta 2 \mathrm{~b}$ and $\beta 3 \mathrm{~d}$ subunits were barely detectable. When compared with the expression levels of the KCa1.1 pore-forming $\alpha$ subunit, the highest relative mRNA expression levels were found for three splice variants of the $\beta 3$ subunit ( $\beta 3 \mathrm{~b}, \beta 3 \mathrm{c}$, and $\beta 3 \mathrm{e}$ ) and for $\beta 4$ subunits (Fig. 1b). Since mRNAs are not always translated into proteins, we used Western blotting to determine protein levels of the different $\beta$ subunits. Analysis of the total cellular protein content showed variable amounts of all $\beta$ subunits of KCa1.1 in different RA-FLS donors compared with the loading control actin. Whereas expression of $\beta 1, \beta 2$, and $\beta 4$ was only detectable in the cell lysates from some donors, $\beta 3$ subunits were consistently identified in all RA-FLS donors but one (Fig. 2).

\section{RA-FLS express either $\beta 1$ or $\beta 3 b$ subunits at their plasma membrane}

The pore-forming $\alpha$ subunits of KCa1.1 can be detected at the plasma membrane and in the nucleus of RA-FLS [11]. Since our focus was on the channels expressed at the plasma membrane of FLS, and since coexpression of $\beta$ subunits with $\alpha$ subunits affects the kinetics and pharmacology of $\mathrm{K}^{+}$currents through the KCa1.1 channel, we used patch-clamp electrophysiology to assess the expression of 
a

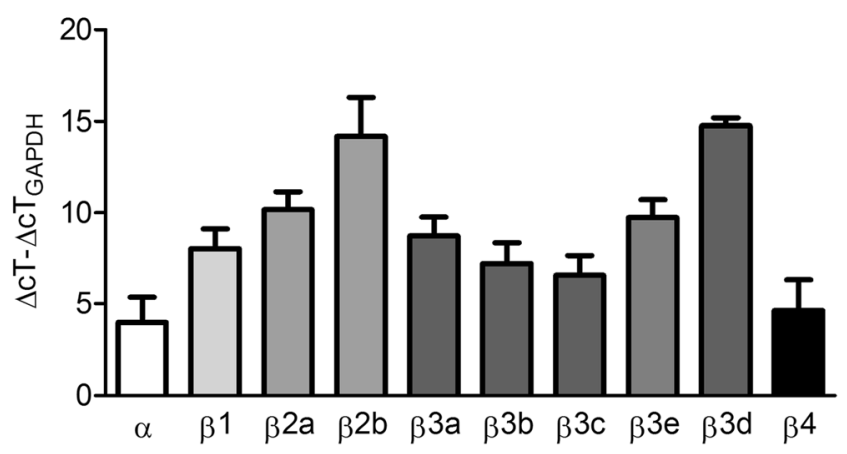

b

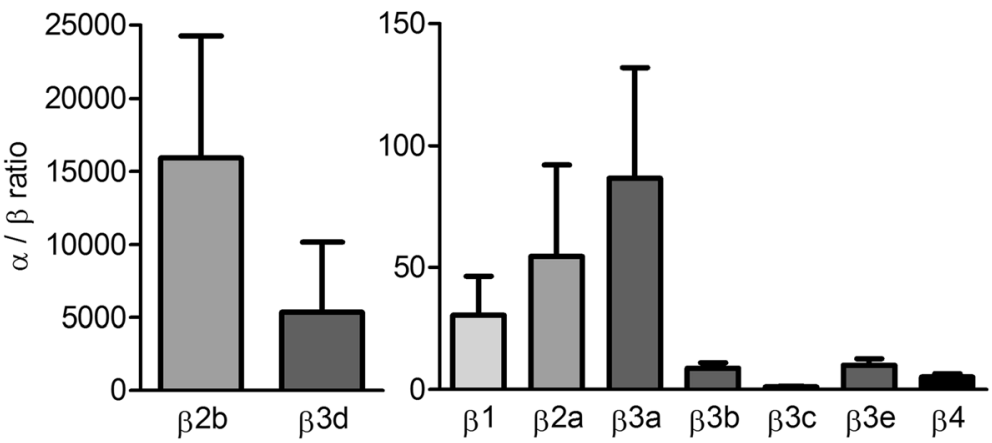

Fig. 1 Fibroblast-like synoviocytes from patients with rheumatoid arthritis express messenger RNA of multiple KCa1.1 $\beta$ subunits. a expression fold measurements were conducted by quantitative reverse transcription polymerase chain reaction, compared with GAPDH expression ( $n=6$ donors with 3 independent repeats). Each bar shows expression of a different KCa1.1 subunit, the letters $a-$-e represent different transcript variants. $\mathbf{b} \alpha / \beta$ ratio showing the amount of KCa1.1 a subunits expressed for each single $\beta$ subunit. Note the different scales of the $y$-axis. $\triangle c T$ comparative cycle threshold, GAPDH glyceraldehyde 3-phosphate dehydrogenase

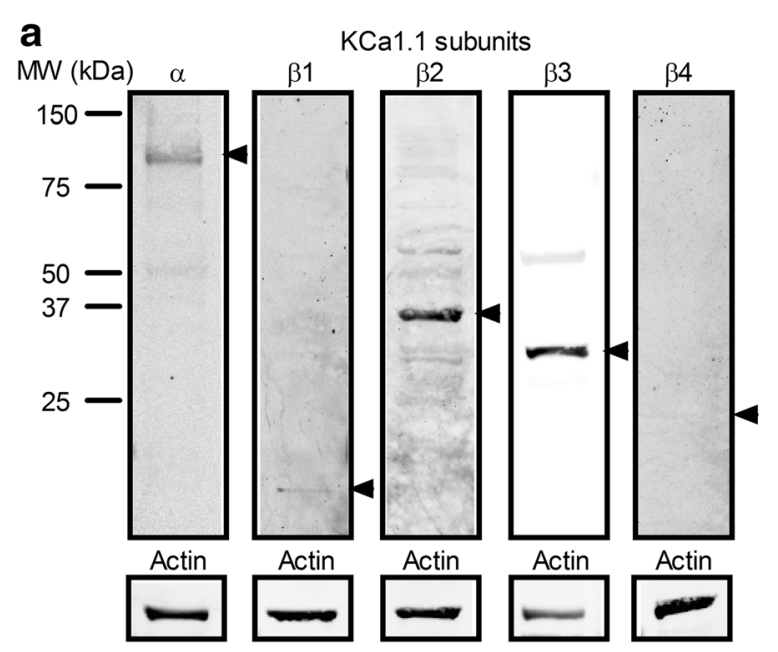

b

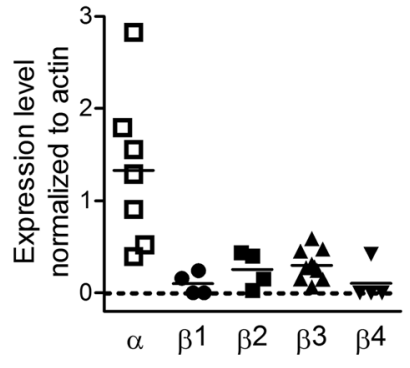

Fig. 2 Fibroblast-like synoviocytes from patients with rheumatoid arthritis (RA-FLS) express proteins of multiple calcium-activated potassium channel KCa1.1 $\beta$ subunits. a Representative Western blot from a gel loaded with proteins from one RA-FLS donor with different lanes probed with antibodies against different subunits of KCa1.1 (top) and actin (bottom). b Intensity of KCa1.1 a and $\beta$ subunit protein bands normalized to actin expression levels in RA-FLS. Each symbol on the scatterplot represents results from a different donor. The horizontal bar represents the mean for each subunit 
functional $\beta$ subunits at the plasma membrane of RA-FLS. Of the 51 cells from 5 different RA-FLS donors patchclamped, 47 (92\%) exhibited a $\mathrm{K}^{+}$current (Fig. 3a), as previously described [11]. Addition of paxilline, a blocker of the KCa1.1 $\alpha$ subunits regardless of $\beta$ subunit expression $[11,14,38]$, completely blocked the $\mathrm{K}^{+}$current in all 14 cells tested (Fig. 3a, b), further confirming that the $\mathrm{K}^{+}$channel observed was KCa1.1, as previously demonstrated [11]. The current displayed little or no inactivation in any of the cells tested (Fig. 3a). Since $\beta 2 a, \beta 3 a, \beta 3 c$, and $\beta 3$ e subunits have all been shown to induce partial or total inactivation of KCa1.1 [36, 39, 45-47], the lack of inactivation shows that these subunits are not involved in the KCa1.1 channel in RA-FLS.

To further identify the $\beta$ subunits associated with the KCa1.1 channels in RA-FLS, we used KCa1.1 openers and blockers known to exert different effects on the channel, depending on its $\beta$ subunit composition. We first tested the effects of the scorpion venom toxin ChTX on RA-FLS $\mathrm{K}^{+}$ currents, as ChTX can block only KCa1.1 channels that do not contain the $\beta 4$ subunit $[21,36,39]$. ChTX inhibited the currents in all cells tested (Fig. 3a, b), demonstrating the absence of $\beta 4$ subunits in KCa1.1 channels of RA-FLS. We next tested the effects of LCA, known to enhance currents through KCa1.1 channels only in the presence of $\beta 1$ subunits [28, 36, 37]. An LCA-induced increase in current was observed in only $36 \%$ of RA-FLS cells tested (Fig. 3a, b), demonstrating that KCa1.1 channels are formed of $\alpha$ and $\beta 1$ subunits in approximately one-third of the cells. To identify the $\beta$ subunit in the remainder of the RA-FLS, we used AA, known to open KCa1.1 channels and thus increase $\mathrm{K}^{+}$currents in the presence of $\beta 2$ and $\beta 3$ subunits,
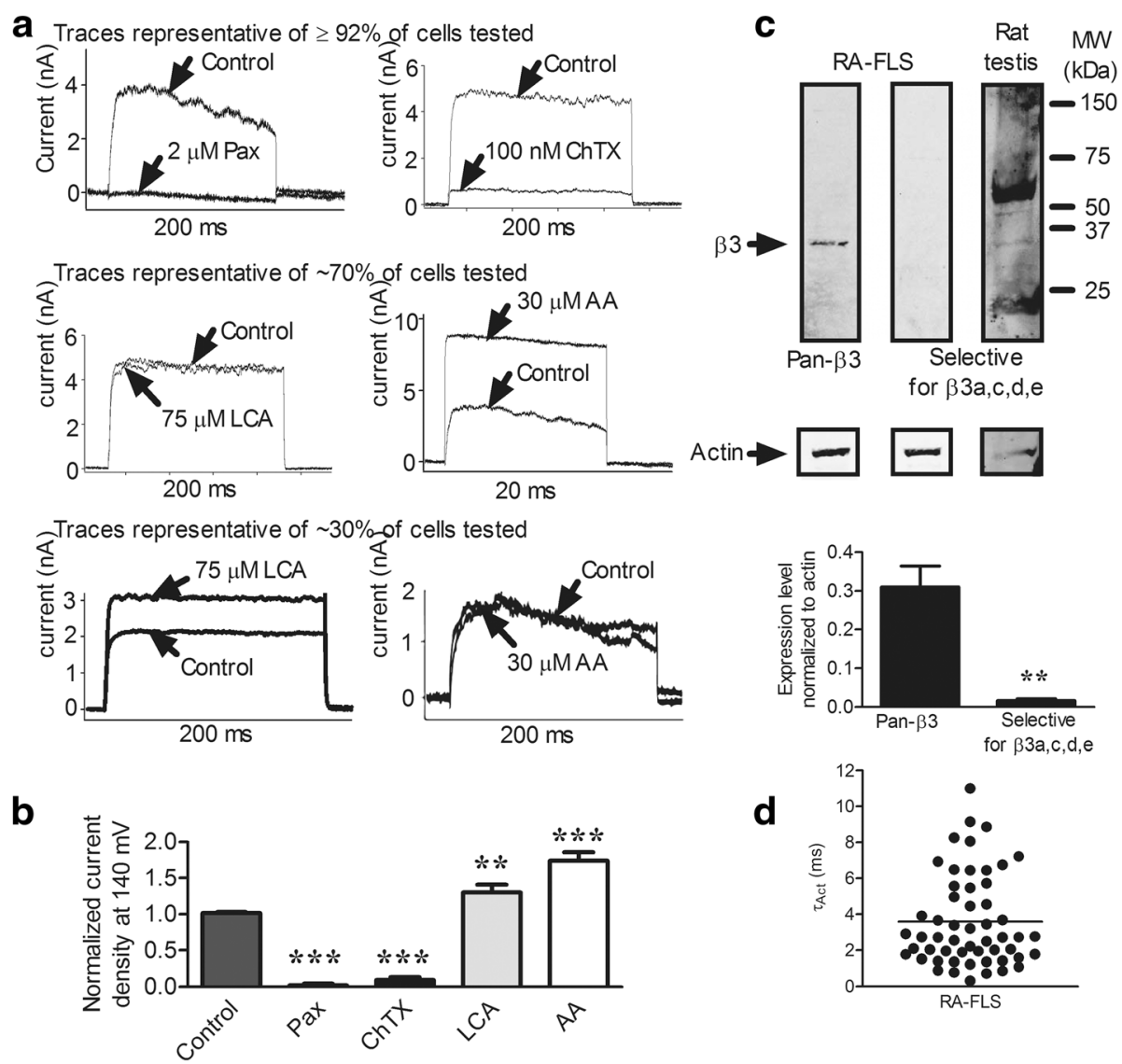

Fig. 3 Functional KCa1.1 $\beta 3$ b subunits are present on the plasma membrane of fibroblast-like synoviocytes from patients with rheumatoid arthritis (RA-FLS). a Representative traces of whole-cell KCa1.1 currents elicited by $140-\mathrm{mV}$ pulses for 200 milliseconds with $5 \mu \mathrm{M} \mathrm{Ca}{ }^{2+}$ in the internal solution before (control) and after applying $2 \mu \mathrm{M}$ paxilline (Pax), $100 \mathrm{nM}$ charybdotoxin (ChTX), $30 \mu \mathrm{M}$ arachidonic acid (AA), or $75 \mu \mathrm{M}$ lithocholic acid (LCA). The two top traces are representative of $\geq 92 \%$ of cells tested; the two middle traces are representative of approximately $70 \%$ of cells tested; and the two bottom traces are representative of the other approximately $30 \%$ of cells tested. b Peak KCa1.1 currents after different treatments normalized to the control levels. Mean \pm SEM; $n=5$ different donors. $\mathbf{c}$ Representative Western blot from a gel loaded with proteins from one RA-FLS donor or a rat testis extract. Different lanes were probed with antibodies against all splice variants of KCa1.1 $\beta 3$ (pan- $\beta 3$ ) or against KCa1.1 $\beta 3 \mathrm{a}, \beta \mathrm{c}$, $\beta \mathrm{d}$, and $\beta$ e only (top), and intensity of KCa1.1 $\beta 3$ protein bands was normalized to actin expression levels in RA-FLS. Mean \pm SEM; $n=6$ different donors. $\mathbf{d}$ Activation kinetics $\left(\tau_{A c t}\right)$ of RA-FLS K ${ }^{+}$currents. Each symbol on the scatterplot represents a different cell. $n=5$ different donors; ${ }^{* *} p \leq 0.01,{ }^{* * *} p \leq 0.001$ 
but not $\beta 1$ or $\beta 4$ subunits $[29,36]$. Such an increase was observed in $65 \%$ of cells tested (Fig. 3a, b). Since the kinetics data above had already eliminated the possibility of $\beta 2 a$, $\beta 3 \mathrm{a}, \beta 3 \mathrm{c}$, or $\beta 3$ e subunits (Fig. 3a), this result with AA suggests that the majority of RA-FLS cells express a $\beta 3$ subunit, either $\beta 3 \mathrm{~b}$ or $\beta 3 \mathrm{~d}$. To discriminate between these two splice variants of $\beta 3$, we performed Western blot experiments using two anti- $\beta 3$ antibodies. The first antibody is directed to a conserved region of the $\beta 3$ subunit, common to all five splice variants, and leads to a band of the correct molecular weight (Figs. 2, 3c). The second antibody used was raised against the $\mathrm{N}$-terminus of $\beta 3$ and therefore detects all splice variants of $\beta 3$ other than $\beta 3 \mathrm{~b}$. Although this antibody detected a band of the correct molecular weight (about $32 \mathrm{kDa}$ ) in rat testis extracts, the only tissue with known $\beta 3$ expression [26], it yielded no detectable band with RA-FLS extracts (Fig. 3c), suggesting that RA-FLS express the $\beta 3 \mathrm{~b}$ subunit of KCa1.1. Since $\beta 1$ but not $\beta 3 \mathrm{~b}$ subunits slow the activation kinetics $\left(\tau_{\mathrm{Act}}\right)$ of $\mathrm{KCa} 1.1$ channels [48], we measured these kinetics in RA-FLS and found a spread in $\tau_{\text {Act }}$ with only 17 (31\%) of the 54 cells assessed having a $\tau_{\text {Act }}$ longer than 4 milliseconds and $69 \%$ of cells having a $\tau_{\text {Act }}$ less than or equal to 4 milliseconds (Fig. 3d). These results reinforce the finding that different individual RA-FLS cells within a line express different $\beta$ subunits.

\section{Expression of $\mathrm{KCa} 1.1 \beta 3 \mathrm{~b}$ is associated with higher levels of KCa1.1a and CD44}

Since invasiveness is an important feature of aggressive FLS during RA, we wanted to test whether invasiveness is associated with a differential expression of KCa1.1 $\beta$ subunits by the cells. In the absence of antibodies that recognize an extracellular epitope of either $\beta 1$ or $\beta 3$ subunits, we searched for a surrogate marker with an extracellular epitope to allow for isolation of live cells. Elevated expression of CD44, a type I transmembrane glycoprotein that binds hyaluronan and other extracellular and cell surface ligands, by FLS and other cells was observed in RA $[49,50]$. Interestingly, elevated CD44 expression is associated with enhanced invasiveness of cancer cells $[51,52]$. To determine whether expression of $\beta 1$ or $\beta 3$ subunits is associated with $\mathrm{CD} 44$ expression levels and invasiveness in RA-FLS, we first showed an association between elevated expression of KCa1.1 $\alpha$ and of CD44 (Fig. 4a). We next used flow cytometry to sort $\mathrm{CD} 44^{\text {high }}$ and $\mathrm{CD} 44^{\text {low }}$ RA-FLS and performed invasion assays. CD $44^{\text {high }}$ RA-FLS were significantly more invasive than $\mathrm{CD} 44^{\text {low }}$ cells (Fig. 4b). Since FLS invasiveness has also been associated with expression of podoplanin, cadherin-11, and MMP-2 [53, 54], we assessed expression levels of these three markers within the $\mathrm{CD} 44^{\text {low }}$ and $\mathrm{CD} 44^{\text {high }}$ populations of RA-FLS. We observed an association between elevated expression of CD44 and all three markers (Fig. 4c). We then stained cells for expression of $\mathrm{CD} 44$ and performed whole-cell patch-clamping to assess $\mathrm{K}^{+}$current densities and $\tau_{\text {Act }}$ in CD $44^{\text {high }}$ and CD $44^{\text {low }}$ cells (Fig. 4d). CD44 $4^{\text {high }}$ cells exhibited higher current densities and faster activation rates than did $C D 44^{\text {low }}$ cells, suggesting expression of $\beta 1$ subunits by $C D 44^{\text {low }}$ cells and of $\beta 3 \mathrm{~b}$ subunits by $\mathrm{CD} 44^{\text {high }}$ cells (Fig. 4e, f). These results were further confirmed by assessing the effects of AA and LCA on the two cell subsets, as $\mathrm{CD} 44^{\text {high }}$ cells displayed sensitivity to AA but not LCA, whereas CD44 ${ }^{\text {low }}$ cells displayed sensitivity to LCA and not AA (Fig. 4g). As a control, we used minimally invasive FLS obtained from patients with OA [55]. OA-FLS exhibited low current densities at $140 \mathrm{mV}$, fast $\tau_{\text {Act }}$, and sensitivity to LCA but not to AA, similar to CD $44^{\text {low }}$ RA-FLS (Fig. 4e-g).

\section{Knocking down the $\beta 3$ subunit of KCa1.1 decreases cell surface expression of the pore-forming a subunit of KCa1.1}

We used a pool of siRNA to selectively inhibit gene expression of the $\beta 3$ subunit. In whole-cell patch-clamp assays, RA-FLS became less sensitive to treatment with the $\beta 3$ opener AA after transfection with $\beta 3$, but not control siRNA, demonstrating the effectiveness of the siRNA. Neither control nor $\beta 3$ siRNA affected the cells' response to LCA, showing a lack of effect on $\beta 1$ subunits (Fig. 5a). Flow cytometry measurements showed that $\beta 3$ silencing induced a $20 \%$ reduction in expression of the KCa1.1 $\alpha$ subunit (Fig. 5b). Moreover, whole-cell KCa1.1 current densities at voltages above $50 \mathrm{mV}$ were significantly decreased after KCa1.1 33 subunit silencing (Fig. 5c), suggesting lower surface expression of the pore-forming $\alpha$ subunit of KCa1.1.

Knocking down the $\beta 3$, but not the $\beta 1$, subunit of $\mathrm{KCa} 1.1$ attenuates the ex vivo invasiveness of RA-FLS

Reducing the expression or function of the $\alpha$ subunit of KCa1.1 inhibits the invasiveness of FLS [11, 12]. We therefore assessed the effects of silencing the $\beta 3$ subunit of KCa1.1 on RA-FLS invasiveness using Matrigel invasion assays. We also used a pool of siRNA to inhibit the expression of the $\beta 1$ subunit of KCa1.1 and used the channel's sensitivity to LCA to demonstrate the effectiveness of the siRNA, as control siRNA did not affect the RA-FLS response to LCA, whereas $\beta 1$ siRNA reduced it (Fig. 6a). Whereas $\beta 3$ siRNA reduced the invasiveness of RA-FLS, silencing the $\beta 1$ subunit of KCa1.1 did not affect this invasiveness (Fig. 6b).

\section{Discussion}

The KCa1.1 channel has been proposed as a therapeutic target in the treatment of RA $[11,12]$. However, the wide tissue distribution of the pore-forming $\alpha$ subunit of the channel precludes the use of blockers targeted to this subunit alone, owing to the risk of severe side 

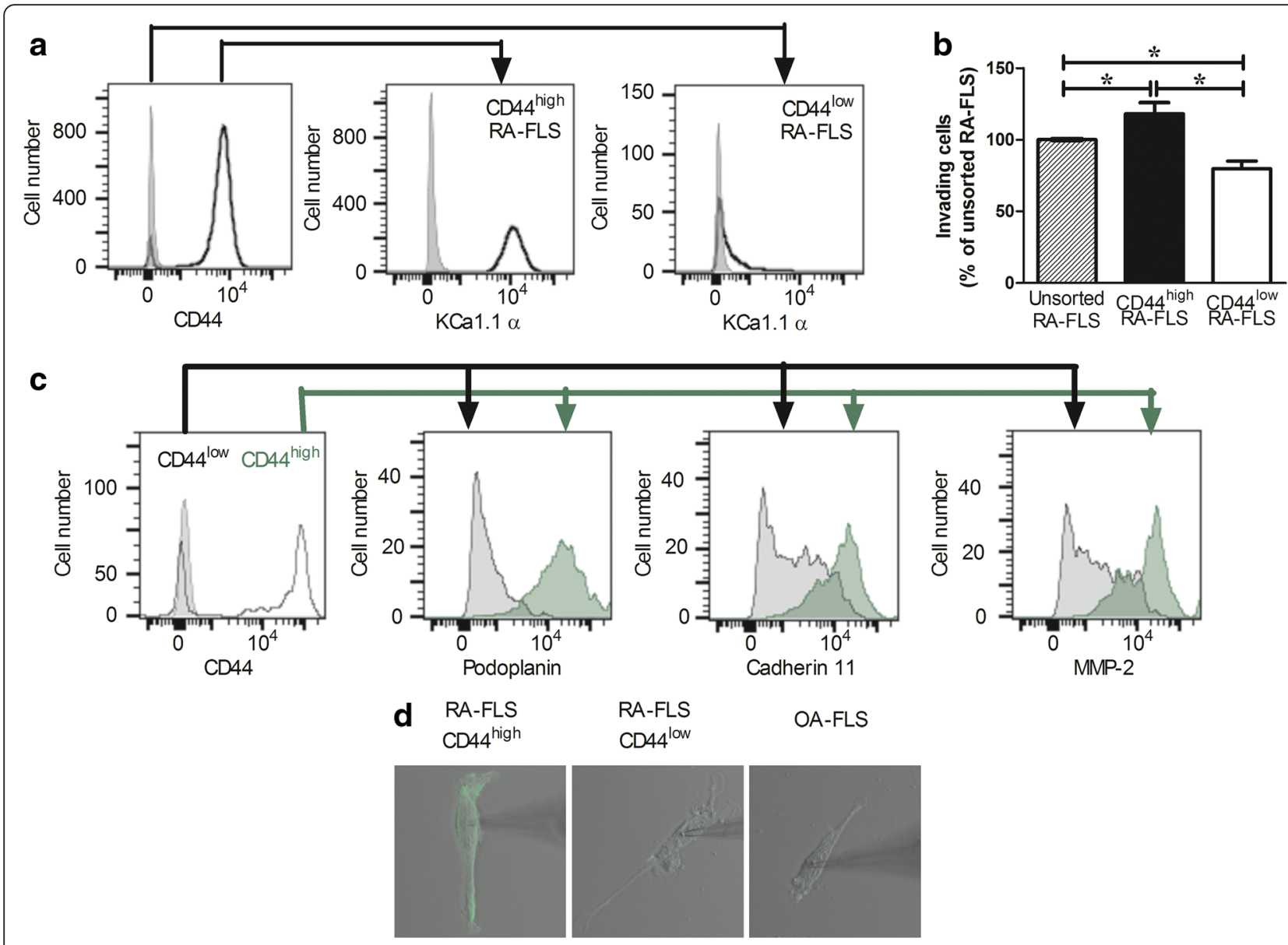

RA-FLS

CD44 $4^{\text {low }}$

OA-FLS
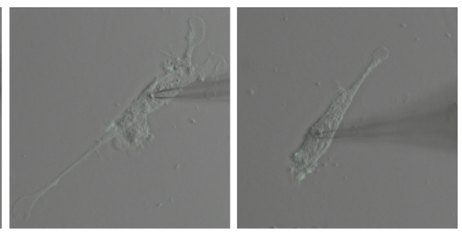

e

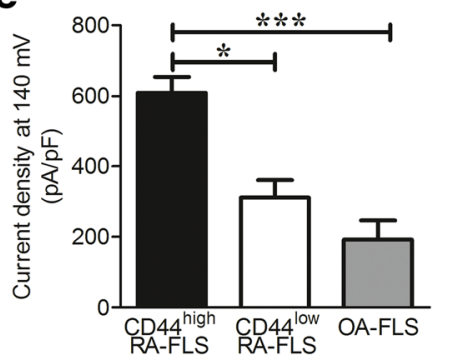

f

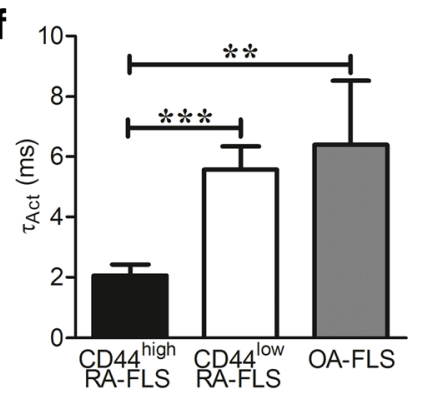

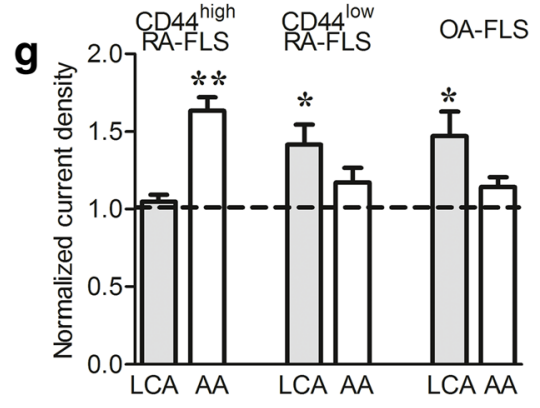

Fig. 4 The calcium-activated potassium channel KCa1.1 $\beta 3$ subunit is expressed by CD44 ${ }^{\text {high }}$ fibroblast-like synoviocytes from patients with rheumatoid arthritis (RA-FLS), whereas the $\beta 1$ subunit is expressed by CD44 ${ }^{\text {low }}$ RA-FLS and fibroblast-like synoviocytes from patients with osteoarthritis (OA-FLS). a Representative flow cytometry histograms showing expression of KCa1.1 a by CD44 ${ }^{\text {high }}$ and CD44 ${ }^{\text {low }}$ RA-FLS. Gray shading represents control staining; black lines represent CD44 (left) or KCa1.1 a (middle and right) staining. $\mathbf{b}$ Invasiveness of unsorted RA-FLS and RA-FLS from the same donors sorted by flow cytometry into CD $44^{\text {low }}$ and CD $44^{\text {high }}$ populations. Mean \pm SEM; $n=3$ RA-FLS donors. The line for the error bar for the control was thickened compared with other plots to make it visible. c Representative flow cytometry histograms showing expression of podoplanin, cadherin-11, and matrix metalloproteinase (MMP)-2 by CD44 ${ }^{\text {high }}$ (green) and CD44 ${ }^{\text {low }}$ (gray) RA-FLS. In the left panel, gray shading represents control staining and black lines represent CD44 staining. $\mathbf{d}$ Individual RA-FLS stained for CD44. The patch-clamp pipette's shadow is visible on the right of each image. $\mathrm{K}^{+}$current density elicited at $140 \mathrm{mV}$ in CD44 ${ }^{\text {high }} \mathrm{RA}-\mathrm{FLS}, \mathrm{CD} 44^{\mathrm{low}} \mathrm{RA}-\mathrm{FLS}$, and OA-FLS. Mean $\pm \mathrm{SEM}$; $n$ $=5$ RA-FLS donors and 4 OA-FLS donors. $\mathbf{f}$ Activation kinetics ( $\left.\tau_{\text {Act }}\right)$ of $\mathrm{K}^{+}$currents elicited at $140 \mathrm{mV}$ in CD44 ${ }^{\text {high }}$ RA-FLS, CD $44^{\text {low }}$ RA-FLS, and OA-FLS. Mean \pm SEM; $n=5$ RA-FLS donors and 4 OA-FLS donors. $g \mathrm{~K}^{+}$current density after treatment of CD44 ${ }^{\text {high }}$ RA-FLS, CD44 ${ }^{\text {low }}$ RA-FLS, and OA-FLS with $75 \mu \mathrm{M}$ lithocholic acid (LCA) or $30 \mu \mathrm{M}$ arachidonic acid (AA) and normalized to current densities before treatment (horizontal dashed line). Mean \pm SEM; $n=5$ RA-FLS donors and 4 OA-FLS donors. ${ }^{*} p \leq 0.05,{ }^{* *} p \leq 0.01,{ }^{* * *} p \leq 0.001$ 


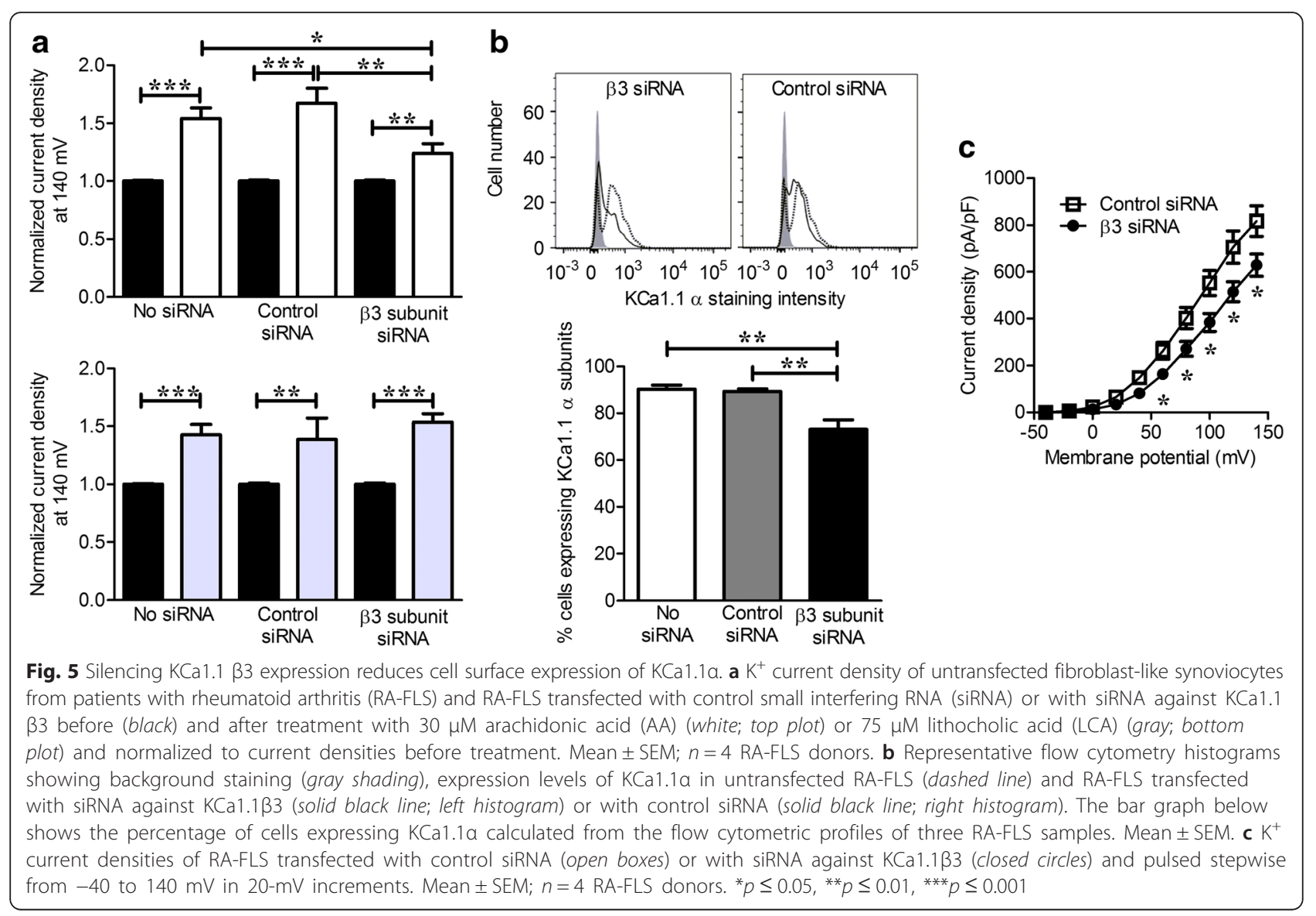

effects in multiple organ systems [16-18]. KCa1.1 does, however, remain an attractive target for therapy because the regulatory $\beta$ subunits of $\mathrm{KCa} 1.1$ have restricted tissue distribution $[20,22]$. In the present study, we demonstrated that RA-FLS express functional $\beta 1$ and $\beta 3$ subunits of $\mathrm{KCa} 1.1$ at their plasma membrane and that expression of $\beta 3$ is higher on CD44 $4^{\text {high }}$ RA-FLS and is associated with a higher expression level of KCa1.1 $\alpha$. Silencing $\beta 3$, but not $\beta 1$, significantly reduced the invasiveness of RA-FLS. In addition, silencing $\beta 3$ reduced the expression level of $\mathrm{KCa} 1.1 \alpha$.

Analysis by qRT-PCR showed expression of most $\beta$ subunits in RA-FLS at the mRNA level. Low mRNA expression levels of $\beta$ accessory subunits have been described in the majority of tissues; therefore, it was not surprising to find similar results in RA-FLS [26]. In addition, mRNAs are not always translated into proteins, requiring the detection of the proteins themselves. In Western blotting experiments, we detected $\beta 3$ subunits in all samples analyzed; however, other $\beta$ subunits were also detectable at the mRNA and protein levels in some samples. Determination of the kinetics and pharmacological profile of KCa1.1 currents of RA-FLS by single-cell electrophysiology demonstrated the expression of functional $\beta 1$ and $\beta 3$ subunits at the plasma membrane. Previous work has also shown the expression of the $\alpha$ subunit of $\mathrm{KCa} 1.1$ in the nucleus of RA-FLS and in several organelles in different cell types [56]. This raises the possibility that $\beta$ subunits of the channel may also be expressed by organelles and play a role not yet understood in FLS.

Levels of the $\beta$ subunits were lower than those of the $\alpha$ subunit as measured at the mRNA and protein levels by qPCR and Western blot analysis, respectively. It is therefore likely that each $\alpha$ subunit tetramer is associated with fewer than four $\beta$ subunits. The ratio of $\beta$ and $\alpha$ subunits required to modify current kinetics and pharmacological responses of KCa1.1 channels remains unclear. Our electrophysiology results demonstrate that the numbers of $\beta 1$ or $\beta 3$ expressed by RA-FLS are sufficient to affect the function of the KCa1.1 channels at their plasma membrane.

The majority of RA-FLS had characteristic whole-cell $\mathrm{KCa} 1.1 \mathrm{~K}^{+}$currents sensitive to paxilline, except for approximately $8 \%$ of the cells that displayed no $\mathrm{K}^{+}$currents under the conditions used, confirming our previous study findings [11]. No single assay is sufficient to identify the $\beta$ subunits associated with $\alpha$ subunits to form KCa1.1 channels. We therefore used a combination of patch-clamp 

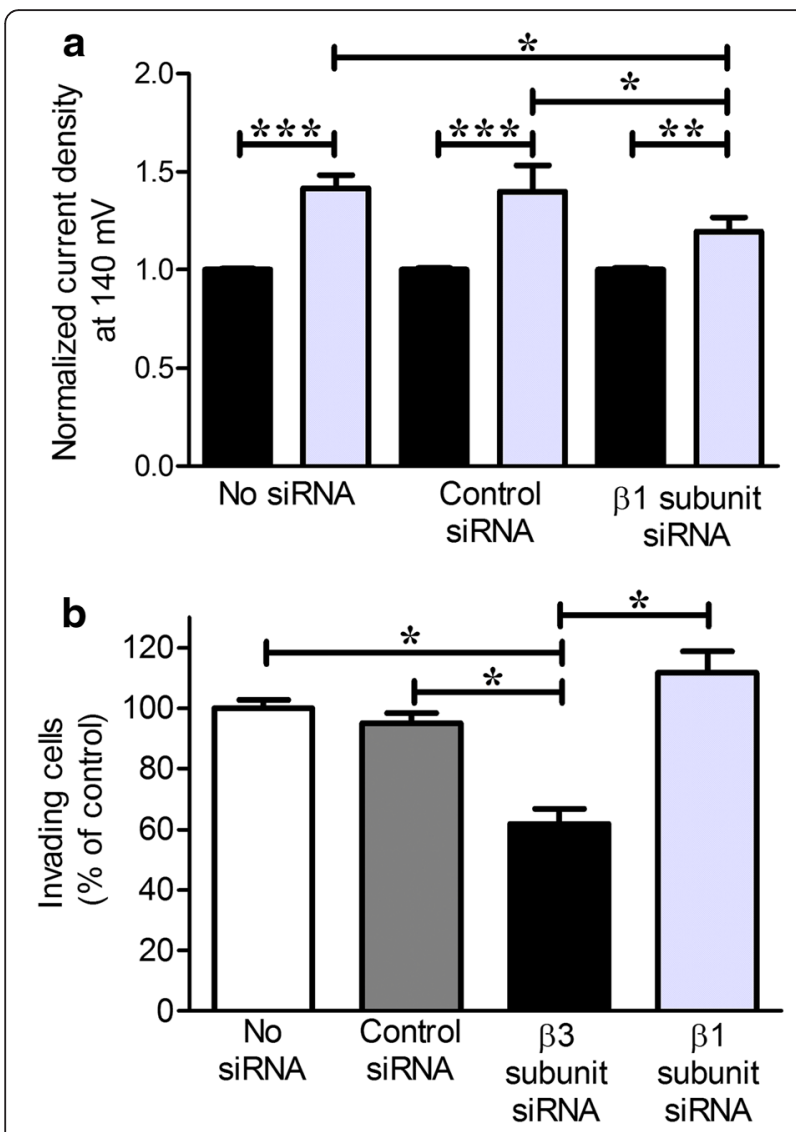

Fig. 6 Silencing calcium-activated potassium channel KCa1.1ß3 but not $\mathrm{KCa} 1.1 \beta 1$ expression reduces inhibits the invasiveness of fibroblast-like synoviocytes from patients with rheumatoid arthritis (RA-FLS). a K ${ }^{+}$current density of untransfected RA-FLS and RA-FLS transfected with control small interfering RNA (siRNA) or with siRNA against KCa1.1 $\beta 1$ before (black) and after treatment with $75 \mu \mathrm{M}$ lithocholic acid (gray) and normalized to current densities before treatment. Mean \pm SEM; $n=3$ RA-FLS donors. $\mathbf{b}$ Invasiveness of untransfected RA-FLS and RA-FLS transfected with control siRNA, with siRNA against KCa1.1 $\beta 3$, or with siRNA against KCa1.1 $\beta 1$. Mean \pm SEM; $n=4$ RA-FLS donors. ${ }^{*} p \leq 0.5,{ }^{* *} p \leq 0.01,{ }^{* * *} p \leq 0.001$

electrophysiology to measure activation and inactivation kinetics of the currents and test the effects of wellcharacterized pharmacological agents and of Western blots using antibodies raised against different epitopes of the $\beta 3$ subunit. When examining the pharmacological response to the perfused agents, we used the bile acid derivate LCA, which, at concentrations of $50-150 \mu \mathrm{M}$ acts as a potent reversible potentiator of KCa1.1 currents only in the presence of $\beta 1$ subunits [27, 28]. A minority of cells responded to LCA, indicating functional $\beta 1$ subunit expression on some RA-FLS. In contrast, treating the RAFLS with $30 \mu \mathrm{M}$ AA, known to enhance KCa1.1 currents only in the presence of $\beta 2$ or $\beta 3$ subunits [29], induced an increase in current amplitude in the majority of the cells. We tested ChTX, which blocks KCa1.1 channels associated with $\beta 1, \beta 2$, and $\beta 3$, but not $\beta 4$ subunits [21], leading to current block in $100 \%$ of the RA-FLS assayed and thus demonstrating the absence of $\beta 4$ subunits as components of KCa1.1 at the plasma membrane of these cells. A phenotype of noninactivating KCa1.1 currents blocked by both paxilline and ChTX, and potentiated by AA but not by LCA, leads to the conclusion that the majority of RAFLS mainly express functional KCa1.1 $\alpha$ and $\beta 3$ subunits. Western blots using antibodies specific to different $\beta 3$ epitopes indicated that RA-FLS express the $\beta 3 \mathrm{~b}$ isoform.

Our data show an association between the expression of KCa1.1 channels formed of $\alpha$ and $\beta 3$ subunits, high current densities, and a high expression of CD44, previously shown in RA synovial tissues $[49,50]$, whereas the $\alpha \beta 1$ phenotype was associated with low expression of CD44 and lower current densities. Since we had previously shown increased expression of $\mathrm{KCa} 1.1 \alpha$ in invasive FLS [12], this raised the possibility that a differential expression of $\beta$ subunits could underlie the different expression levels of the $\alpha$ subunit of KCa1.1. Indeed, silencing of the $\beta 3$ subunit did decrease expression levels of $\mathrm{KCa} 1.1 \alpha$ and reduced the $\mathrm{K}^{+}$current densities, suggesting that $\beta 3$ may participate in the cell surface expression of the channel, as has been described in other systems with $\beta 1, \beta 2$, and $\beta 4[23,24,30]$.

To our knowledge, an association between high expression levels of a potassium channels and CD44 has not directly been reported. However, CD44 expression has long been associated with cancer cell metastasis [52]. More recently, the expression of various potassium channels, including $\mathrm{KCa} 1.1$, has also been linked to enhanced metastatic potential in cancer [57]. Further work needs to be done to determine whether CD44 and potassium channels share any signaling pathways leading to their concomitant upregulation in highly motile cells and to assess whether their expression or function is interdependent in these cells. Additional work is also required to determine whether the switch from KCa1.1 $\beta 1$ to $\beta 3 \mathrm{~b}$ expression in RA-FLS is a consequence, an initiating event, or an independent event in the upregulation of CD44 by these cells. Finally, it will be interesting to establish whether $\mathrm{KCa} 1.1$ and $\mathrm{CD} 44$ play synergistic roles in regulating the invasiveness of RA-FLS.

The work presented here was focused on the regulatory $\beta$ subunits of $\mathrm{KCa1}$.1. In the last few years, $\gamma$ subunits of $\mathrm{KCa} 1.1$ have also been identified in various tissues $[25,58]$. These subunits are structurally different from $\beta$ subunits, but, like $\beta$ subunits, they affect the function of the KCa1.1 channels with which they are coexpressed. It is possible that FLS express $\gamma$ subunits of KCa1.1 in addition to the $\beta$ subunits we have identified, either at the plasma membrane or in organelles.

The KCa1.1 channel formed of $\alpha$ and $\beta 3$ subunits expressed by RA-FLS represents an attractive 
therapeutic target for RA. The mRNA for $\beta 3$ is expressed only in very low quantity in most tissues, with the highest levels detected in the testis [26]. The incidence of RA is higher in women than in men [1-3]; in the majority of patients, there would therefore not be a concern about male reproductive organ toxicity. Furthermore, the testes are protected by the blood-testis barrier that prevents access by many drugs [59]. It is therefore possible to design KCa1.1 blockers that cannot cross this barrier.

In addition to this restricted tissue distribution, the presence of $\beta$ subunits alters the pharmacology of the channel. Indeed, several scorpion venom peptides affect KCa1.1 channels differently, depending on the $\beta$ subunit expressed. ChTX and iberiotoxin both block KCa1.1 channels containing $\beta 1, \beta 2$, and $\beta 3$ subunits, but not $\beta 4$ subunits, $[21,60]$. Martentoxin blocks KCa1.1 formed of $\alpha \beta 4$, whereas it opens KCa1.1 formed of $\alpha \beta 1$ and has yet to be tested on KCa1.1 containing $\beta 2$ or $\beta 3$ subunits [61]. It is therefore conceivable that venom peptides that selectively target KCa1.1 $\alpha \beta 3$ could be identified or that existing KCa1.1blocking peptides could be engineered to enhance their selectivity for this channel as was done successfully with other $\mathrm{K}^{+}$channel-blocking peptides [62-64].

\section{Conclusions}

We provide evidence that the KCa1.1 channels expressed at the plasma membrane of human FLS contain regulatory $\beta 1$ or $\beta 3 \mathrm{~b}$ subunits. Expression of the $\beta 3 \mathrm{~b}$ subunit is associated with a highly invasive behavior of FLS and high expression of CD44, whereas the $\beta 1$ subunit is associated with low levels of CD44 and lower invasiveness of the cells. These data suggest that a blocker selective for KCa1.1 channels formed of $\alpha$ and $\beta 3 \mathrm{~b}$ subunits and that cannot cross the blood-testis barrier will be attractive for targeting KCa1.1 channels on invasive RA-FLS for the treatment of RA.

\begin{abstract}
Abbreviations
AA: arachidonic acid; cDNA: complementary DNA; ChTX: charybdotoxin; $\Delta c T$ : comparative cycle threshold; DMARD: disease-modifying antirheumatic drug; DMSO: dimethyl sulfoxide; EP: electrophysiology; FC: flow cytometry; FITDP: Feinstein Institute Tissue Donation Program; FLS: fibroblast-like synoviocyte(s); GAPDH: glyceraldehyde 3-phosphate dehydrogenase; IgG: immunoglobulin G; IRB: institutional review board; KCa: calciumactivated potassium channel; LCA: lithocholic acid; MMP: matrix metalloproteinase; mRNA: messenger RNA; N/A: not available; NSAID: nonsteroidal anti-inflammatory drug; OA: osteoarthritis; OA-FLS: fibroblast-like synoviocytes from patients with osteoarthritis; RA: rheumatoid arthritis; RA-FLS: fibroblast-like synoviocytes from patients with rheumatoid arthritis; RF: rheumatoid factor; RT: reverse transcription; siRNA: small interfering RNA; $\tau_{\text {Act: }}$ activation kinetics; WB: Western blot.
\end{abstract}

\section{Competing interests}

The authors declare that they have no competing interests.

\section{Authors' contributions}

ZP and RBT performed the RNA and protein extractions, the qPCRs, and Western blotting. ZP, RH, and CB performed the electrophysiology experiments. ZP, MRT, and CB performed the flow cytometry assays. ZP and MRT conducted the invasion assays. ZP and MRT performed the assays with siRNAs. TL and PSG maintained the FLS and verified their purity. GP provided expertise and critical feedback. ZP and CB wrote the manuscript with input from all authors. All authors read and approved the final manuscript.

\section{Acknowledgements}

This work was supported by funding from the Baylor College of Medicine (to C.B.). Z.P. was supported by a fellowship from the Campus Hungary Association. M.R.T. was supported in part by National Institutes of Health awards GM088129 and AI053831. R.H. was supported in part by National Institutes of Health award HL007676. The Cytometry and Cell Sorting Core at Baylor College of Medicine is supported in part by National Institutes of Health grants RR024574, AI036211, and CA125123.

\section{Author details}

${ }^{1}$ Department of Molecular Physiology and Biophysics, Mail Stop BCM335, Room S409A, Baylor College of Medicine, Houston, TX 77030, USA. ${ }^{2}$ Department of Biophysics and Cell Biology, Faculty of Medicine, University of Debrecen, Debrecen 4032, Hungary. ${ }^{3}$ Interdepartmental Graduate Program in Translational Biology and Molecular Medicine, Baylor College of Medicine, Houston, TX 77030, USA. ${ }^{4}$ Graduate Program in Molecular Physiology and Biophysics, Baylor College of Medicine, Houston, TX 77030, USA. ${ }^{5}$ Division of Rheumatology, Icahn School of Medicine at Mount Sinai, New York, NY 10029, USA. 'Biology of Inflammation Center, Baylor College of Medicine, Houston, TX 77030, USA. ${ }^{7}$ Center for Drug Discovery, Baylor College of Medicine, Houston, TX 77030, USA.

Received: 27 December 2015 Accepted: 26 April 2016

Published online: 10 May 2016

\section{References}

1. Gregersen PK, Plenge RM, Gulko PS. Genetics of rheumatoid arthritis. In: Firestein GS, Panayi GS, Wollheim FA, editors. Rheumatoid arthritis. 2nd ed. New York: Oxford University Press; 2006. p. 3-14.

2. Gulko PS, Winchester RJ. Rheumatoid arthritis. In: Austen KF, Frank MM, Atkinson JP, Cantor H, editors. Samter's immunologic diseases. 6th ed. Philadelphia: Lippincott Williams \& Wilkins; 2001. p. 427-63.

3. Gibofsky A. Overview of epidemiology, pathophysiology, and diagnosis of rheumatoid arthritis. Am J Manag Care. 2012;18(13 Suppl):S295-302.

4. Kahlenberg JM, Fox DA. Advances in the medical treatment of rheumatoid arthritis. Hand Clin. 2011;27(1):11-20.

5. Fidder $\mathrm{HH}$, Singendonk MM, van der Have M, Oldenburg B, van Oijen MG. Low rates of adherence for tumor necrosis factor-a inhibitors in Crohn's disease and rheumatoid arthritis: results of a systematic review. World J Gastroenterol. 2013;19:4344-450.

6. Noss EH, Brenner MB. The role and therapeutic implications of fibroblast-like synoviocytes in inflammation and cartilage erosion in rheumatoid arthritis. Immunol Rev. 2008:223:252-70.

7. Bartok B, Firestein GS. Fibroblast-like synoviocytes: key effector cells in rheumatoid arthritis. Immunol Rev. 2010;233:233-55.

8. Bottini N, Firestein GS. Duality of fibroblast-like synoviocytes in RA: passive responders and imprinted aggressors. Nat Rev Rheumatol. 2013;9:24-33.

9. Laragione T, Brenner M, Mello A, Symons M, Gulko PS. The arthritis severity locus Cia5d is a novel genetic regulator of the invasive properties of synovial fibroblasts. Arthritis Rheum. 2008;58:2296-306.

10. Tolboom TC, van der Helm-Van Mil AH, Nelissen RG, Breedveld FC, Toes RE, Huizinga TW. Invasiveness of fibroblast-like synoviocytes is an individual patient characteristic associated with the rate of joint destruction in patients with rheumatoid arthritis. Arthritis Rheum. 2005:52:1999-2002.

11. Hu X, Laragione T, Sun L, Koshy S, Jones KR, Ismailov II, et al. KCa1.1 potassium channels regulate key pro-inflammatory and invasive properties of fibroblastlike synoviocytes in rheumatoid arthritis. J Biol Chem. 2012;287:4014-22.

12. Tanner MR, Hu X, Huq R, Tajhya RB, Sun L, Khan FS, et al. KCa1.1 inhibition attenuates fibroblast-like synoviocyte invasiveness and ameliorates rat models of rheumatoid arthritis. Arthritis Rheumatol 2015;67:96-106

13. Saleem F, Rowe ICM, Shipston MJ. Characterization of BK channel splice variants using membrane potential dyes. Br J Pharmacol. 2009;156:143-52. 
14. Zhou Y, Lingle CJ. Paxilline inhibits BK channels by an almost exclusively closed-channel block mechanism. J Gen Physiol. 2014;144:415-40.

15. Sanchez M, McManus OB. Paxilline inhibition of the alpha-subunit of the high-conductance calcium-activated potassium channel. Neuropharmacology. 1996;35(7):963-8.

16. Meredith AL, Thorneloe KS, Werner ME, Nelson MT, Aldrich RW. Overactive bladder and incontinence in the absence of the BK large conductance $\mathrm{Ca}^{2}$ ${ }^{+}$-activated K+ channel. J Biol Chem. 2004;279:36746-52.

17. Sausbier M, Arntz C, Bucurenciu I, Zhao H, Zhou XB, Sausbier U, et al. Elevated blood pressure linked to primary hyperaldosteronism and impaired vasodilation in BK channel-deficient mice. Circulation. 2005;112:60-8.

18. Sausbier M, Hu H, Arntz C, Feil S, Kamm S, Adelsberger H, et al. Cerebellar ataxia and Purkinje cell dysfunction caused by $\mathrm{Ca}^{2+}$-activated $\mathrm{K}^{+}$channel deficiency. Proc Natl Acad Sci U S A. 2004;101:9474-8.

19. Contreras GF, Neely A, Alvarez O, Gonzalez C, Latorre R. Modulation of BK channel voltage gating by different auxiliary $\beta$ subunits. Proc Natl Acad Sci U S A. 2012;109:18991-6.

20. Brenner R, Jegla TJ, Wickenden A, Liu Y, Aldrich RW. Cloning and functional characterization of novel large conductance calcium-activated potassium channel $\beta$ subunits, hKCNMB3 and hKCNMB4. J Biol Chem. 2000:275:6453-61.

21. Meera $P$, Wallner $M$, Toro L. A neuronal beta subunit (KCNMB4) makes the large conductance, voltage- and $\mathrm{Ca}^{2+}$-activated $\mathrm{K}^{+}$channel resistant to charybdotoxin and iberiotoxin. Proc Natl Acad Sci U S A. 2000;97:5562-7.

22. Orio $P$, Rojas $P$, Ferreira $G$, Latorre R. New disguises for an old channel: MaxiK channel $\beta$-subunits. News Physiol Sci. 2002;17:156-61.

23. Shruti S, Urban-Ciecko J, Fitzpatrick JA, Brenner R, Bruchez MP, Barth AL. The brain-specific $\beta 4$ subunit downregulates BK channel cell surface expression. PLoS One. 2012;7, e33429.

24. Toro B, Cox N, Wilson RJ, Garrido-Sanabria E, Stefani E, Toro L, et al. KCNMB1 regulates surface expression of a voltage and $\mathrm{Ca}^{2+}$-activated $\mathrm{K}^{+}$channel via endocytic trafficking signals. Neuroscience. 2006;142:661-9.

25. Yan J, Aldrich RW. BK potassium channel modulation by leucine-rich repeatcontaining proteins. Proc Natl Acad Sci U S A. 2012;109:7917-22.

26. Behrens $R$, Nolting A, Reimann F, Schwarz M, Waldschutz R, Pongs $O$. hKCNMB3 and hKCNMB4, cloning and characterization of two members of the large-conductance calcium-activated potassium channel $\beta$ subunit family. FEBS Lett. 2000;474(1):99-106.

27. Bukiya AN, McMillan J, Parrill AL, Dopico AM. Structural determinants of monohydroxylated bile acids to activate $\beta_{1}$ subunit-containing BK channels. J Lipid Res. 2008;49(11):2441-51.

28. Bukiya AN, Vaithianathan T, Toro L, Dopico AM. Channel $\beta 2-4$ subunits fail to substitute for $\beta 1$ in sensitizing BK channels to lithocholate. Biochem Biophys Res Commun. 2009;390:995-1000.

29. Sun X, Zhou D, Zhang P, Moczydlowski EG, Haddad GG. $\beta$-subunitdependent modulation of $h$ Slo BK current by arachidonic acid. J Neurophysiol. 2007;97:62-9.

30. Zarei MM, Song M, Wilson RJ, Cox N, Colom LV, Knaus HG, et al. Endocytic trafficking signals in KCNMB2 regulate surface expression of a large conductance voltage and $\mathrm{Ca}^{2+}$-activated $\mathrm{K}^{+}$channel. Neuroscience. 2007;147:80-9.

31. Aletaha D, Neogi T, Silman AJ, Funovits J, Felson DT, Bingham CO, et al. 2010 Rheumatoid arthritis classification criteria: an American College of Rheumatology/European League Against Rheumatism collaborative initiative. Arthritis Rheum. 2010;62:2569-81.

32. Chan A, Akhtar M, Brenner M, Zheng Y, Gulko PS, Symons M. The GTPase Rac regulates the proliferation and invasion of fibroblast-like synoviocytes from rheumatoid arthritis patients. Mol Med. 2007;13:297-304.

33. Laragione T, Gulko PS. mTOR regulates the invasive properties of synovial fibroblasts in rheumatoid arthritis. Mol Med. 2010;16:352-8.

34. Wulff H, Calabresi P, Allie R, Yun S, Pennington MW, Beeton C, et al. The voltage-gated $\mathrm{Kv} 1.3 \mathrm{~K}^{+}$channel in effector memory $T$ cells as new target for MS. J Clin Invest. 2003;111:1703-13.

35. Beeton C, Pennington MW, Wulff $\mathrm{H}$, Singh $\mathrm{S}$, Nugent $\mathrm{D}$, Crossley $\mathrm{G}$, et al. Targeting effector memory T cells with a selective peptide inhibitor of Kv1.3 channels for therapy of autoimmune diseases. Mol Pharmacol. 2005;67:1369-81.

36. Torres YP, Granados ST, Latorre R. Pharmacological consequences of the coexpression of BK channels a and $\beta$ subunits. Front Physiol. 2014;5:383.

37. Bukiya AN, Liu J, Toro L, Dopico AM. $\beta_{1}$ (KCNMB1) subunits mediate lithocholate activation of large-conductance $\mathrm{Ca}^{2+}$-activated $\mathrm{K}^{+}$channels and dilation in small, resistance-size arteries. Mol Pharmacol. 2007;72:359-69.
38. Strøbaek D, Christophersen P, Holm NR, Moldt P, Ahring PK, Johansen TE, et al. Modulation of the $\mathrm{Ca}^{2+}$-dependent $\mathrm{K}^{+}$channel, hslo, by the substituted diphenylurea NS 1608, paxilline and internal $\mathrm{Ca}^{2+}$. Neuropharmacology. 1996;35(7):903-14.

39. Wang B, Jaffe DB, Brenner R. Current understanding of iberiotoxin-resistant BK channels in the nervous system. Front Physiol. 2014;5:382.

40. Chhabra S, Chang SC, Nguyen HM, Huq R, Tanner MR, Londono LM, et al. Kv1.3 channel-blocking immunomodulatory peptides from parasitic worms: implications for autoimmune diseases. FASEB J. 2014;28:3952-64.

41. Koshy S, Huq R, Tanner MR, Atik MA, Porter PC, Khan FS, et al. Blocking Kv1. 3 channels inhibits Th2 lymphocyte function and treats a rat model of asthma. J Biol Chem. 2014;289:12623-32.

42. Tarcha EJ, Chi V, Munoz-Elias E, Bailey D, Londono LM, Upadhyay SK, et al. Durable pharmacological responses from the peptide ShK-186, a specific Kv1.3 channel inhibitor that suppresses T cell mediators of autoimmune diseases. J Pharmacol Exp Ther. 2012;342:642-53.

43. Beeton C, Wulff H, Singh S, Botsko S, Crossley G, Gutman GA, et al. A novel fluorescent toxin to detect and investigate Kv1.3 channel upregulation in chronically activated T lymphocytes. J Biol Chem. 2003;278(11):9928-37.

44. Laragione T, Cheng KF, Tanner MR, He M, Beeton C, Al-Abed Y, et al. The cation channel Trpv2 is a new suppressor of arthritis severity, joint damage, and synovial fibroblast invasion. Clin Immunol. 2015;158:183-92.

45. Hu S, Labuda MZ, Pandolfo M, Goss GG, McDermid HE, Ali DW. Variants of the KCNMB3 regulatory subunit of maxi BK channels affect channel inactivation. Physiol Genomics. 2003;15:191-8.

46. Orio $P$, Latorre R. Differential effects of $\beta 1$ and $\beta 2$ subunits on BK channel activity. J Gen Physiol. 2005;125:395-411.

47. Wallner M, Meera P, Toro L. Molecular basis of fast inactivation in voltage and $\mathrm{Ca}^{2+}$-activated $\mathrm{K}^{+}$channels: a transmembrane $\beta$-subunit homolog. Proc Natl Acad Sci U S A. 1999;96(7):4137-42.

48. Lee US, Cui J. $\beta$ subunit-specific modulations of BK channel function by a mutation associated with epilepsy and dyskinesia. J Physiol. 2009:587:1481-98.

49. Haynes BF, Hale LP, Patton KL, Martin ME, McCallum RM. Measurement of an adhesion molecule as an indicator of inflammatory disease activity: upregulation of the receptor for hyaluronate (CD44) in rheumatoid arthritis. Arthritis Rheum. 1991;34:1434-43.

50. Naor D, Nedvetzki S. CD44 in rheumatoid arthritis. Arthritis Res Ther. 2003:5:105-15.

51. Knudson W. The role of CD44 as a cell surface hyaluronan receptor during tumor invasion of connective tissue. Front Biosci. 1998:3:d604-15.

52. Jothy S. CD44 and its partners in metastasis. Clin Exp Metastasis. 2003;20:195-201.

53. Kiener HP, Niederreiter B, Lee DM, Jimenez-Boj E, Smolen JS, Brenner MB. Cadherin 11 promotes invasive behavior of fibroblast-like synoviocytes. Arthritis Rheum. 2009;60(5):1305-10.

54. Ekwall AK, Eisler T, Anderberg C, Jin C, Karlsson N, Brisslert M, et al. The tumour-associated glycoprotein podoplanin is expressed in fibroblast-like synoviocytes of the hyperplastic synovial lining layer in rheumatoid arthritis. Arthritis Res Ther. 2011;13(2):R40.

55. Tolboom TCA, Pieterman E, van der Laan WH, Toes REM, Huidekoper AL, Nelissen RGHH, et al. Invasive properties of fibroblast-like synoviocytes: correlation with growth characteristics and expression of MMP-1, MMP-3, and MMP-10. Ann Rheum Dis. 2002;61:975-80.

56. Singh $H_{\text {, Stefani }}$, Toro L. Intracellular BK $\mathrm{Ca}_{\mathrm{Ca}}$ (iBK $\mathrm{Ca}_{\mathrm{a}}$ ) channels. J Physiol. 2012; 590:5937-47.

57. Comes N, Serrano-Albarrás A, Capera J, Serrano-Novillo C, Condom E, Ramón $Y$, et al. Involvement of potassium channels in the progression of cancer to a more malignant phenotype. Biochim Biophys Acta. 1848;2015:2477-92

58. Zhang J, Yan J. Regulation of BK channels by auxiliary $y$ subunits. Front Physiol. 2014;5:401.

59. Quignot N. Modeling bioavailability to organs protected by biological barriers. In Silico Pharmacol. 2013;1:8.

60. Candia S, Garcia ML, Latorre R. Mode of action of iberiotoxin, a potent blocker of the large conductance Ca ${ }^{2+}$-activated K+ channel. Biophys J. 1992;63:583-90.

61. Tao J, Shi J, Liu ZR, Ji YH. Martentoxin: a unique ligand of BK channels. Sheng Li Xue Bao. 2012;64:355-64.

62. Beeton C, Pennington MW, Norton RS. Analogs of the sea anemone potassium channel blocker ShK for the treatment of autoimmune diseases. Inflamm Allergy Drug Targets. 2011;10:313-21. 
63. Beeton C. Targets and therapeutic properties. In: Kastin AJ, editor. Handbook of biologically active peptides. 2nd ed. San Diego, CA: Elsevier/Academic Press; 2013. p. 473-82.

64. Chi V, Pennington MW, Norton RS, Tarcha EJ, Londono LM, Sims-Fahey B, et al. Development of a sea anemone toxin as an immunomodulator for therapy of autoimmune diseases. Toxicon. 2012;59:529-46.

Submit your next manuscript to BioMed Central and we will help you at every step:

- We accept pre-submission inquiries

- Our selector tool helps you to find the most relevant journal

- We provide round the clock customer support

- Convenient online submission

- Thorough peer review

- Inclusion in PubMed and all major indexing services

- Maximum visibility for your research

Submit your manuscript at www.biomedcentral.com/submit 